\title{
Erratum: Optical selection rules of zigzag graphene nanoribbons [Phys. Rev. B 95, 155438 (2017)]
}

\author{
V. A. Saroka, M. V. Shuba, and M. E. Portnoi \\ (Received 27 October 2017; published 10 November 2017)
}

DOI: 10.1103/PhysRevB.96.199901

We have spotted four typographical errors in the original paper which may affect its understanding. First, in the caption for Fig. 2 the values of the parameter $q=2 \cos (k / 2)$ are to be assigned to the figure panels in the reverse order. Thus, the correct values of the parameter $q=2 \cos (k / 2)$ in the panels for Fig. 2 are (a) 2 , (b) $3 w / 2(w+1)$, (c) $w /(w+1)$, and (d) 0 . Second, in the caption for Fig. 3 the following part "and the Brillouin zone points $k=k_{t}+\delta$ :(a) $\delta=-0.3$, (b) 0 , and (c) 0.3 ." should be read as "and the Brillouin zone points $k=k_{t}+i \delta: \delta=0.3$ and (a) $i=-1$, (b) 0 , and (c) 1." Third, the absorption spectra presented in Figs. 8 and 9 are not normalized by the number of atoms in the structure unit cell. Therefore, the sentence on p. 11: "For the sake of comparison, each spectrum is normalized by the number of atoms in the unit cell." should be read, in fact, as "For the sake of comparison, each spectrum is not normalized by the number of atoms in the unit cell." The discussion of the figures remains the same. Finally, in Appendix E in the caption for Fig. 11 the temperatures are expressed in the units of K/ $\gamma$. Thus, we have temperatures: $T=0,4,77$, and $300 \mathrm{~K} / \gamma$ for curves (1), (2), (3), and (4), respectively.

All the above-mentioned corrections do not affect the main conclusions of the paper. 